\title{
Field Portable Low Temperature Porous Layer Open Tubular Cryoadsorption Headspace Sampling and Analysis Part II: Applications*
}

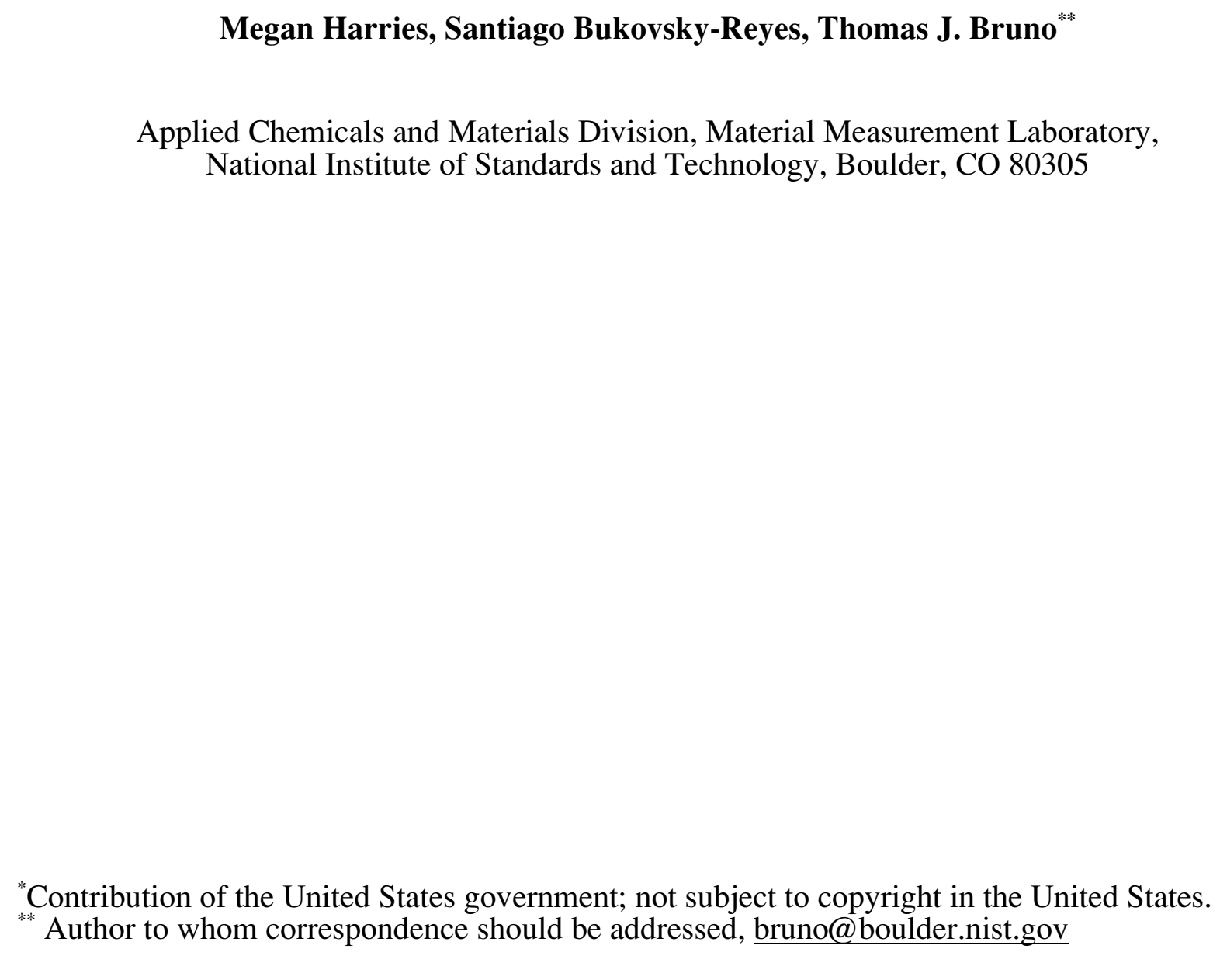

Megan Harries, Santiago Bukovsky-Reyes, Thomas J. Bruno***

Applied Chemicals and Materials Division, Material Measurement Laboratory, National Institute of Standards and Technology, Boulder, CO 80305

*Contribution of the United States government; not subject to copyright in the United States.

** Author to whom correspondence should be addressed, bruno@boulder.nist.gov 
This paper details the sampling methods used with the field portable porous layer open tubular cryoadsorption (PLOT-cryo) approach, described in Part I of this two-part series, applied to several analytes of interest. We conducted tests with coumarin and 2,4,6-trinitrotoluene (two solutes that were used in initial development of PLOT-cryo technology), naphthalene, aviation turbine kerosene, and diesel fuel, on a variety of matrices and test beds. We demonstrated that these analytes can be easily detected and reliably identified using the portable unit for analyte collection. By leveraging efficiency-boosting temperature control and the high flow rate multiple capillary wafer, very short collection times (as low as $3 \mathrm{~s}$ ) yielded accurate detection. For diesel fuel spiked on glass beads, we determined a method detection limit below $1 \mathrm{ppm}$. We observed greater variability among separate samples analyzed with the portable unit than previously documented in work using the laboratory-based PLOT-cryo technology. We identify three likely sources that may help explain the additional variation: the use of a compressed air source to generate suction, matrix geometry, and variability in the local vapor concentration around the sampling probe as solute depletion occurs both locally around the probe and in the test bed as a whole. This field-portable adaptation of the PLOT-cryo approach has numerous and diverse potential applications. 


\subsection{Introduction}

This paper is Part II of a two-part series on field portable porous layer open tubular cryoadsorption (PLOT-cryo). Part I describes the apparatus and sampling method. [1] We anticipate the utility of the field portable approach in a multitude of applications, for example, environmental and criminalistic sampling situations. This might include sampling pollution due to illegal dumping, leaks in fracking wells and service tanks, the location of clandestine graves, the detection of food spoilage, and arson fire debris investigation. In previous work, we have applied the laboratory-based PLOT-cryo approach to several of these applications. [2-5]

The field portable method and apparatus was applied to the vapor analysis of coumarin, 2,4,6trinitrotoluene (2-methyl-1,3,5-trinitrobenzene, TNT), aviation turbine fuel, and naphthalene. These samples are four of the benchmark analytes used in previous work to evaluate the performance of PLOT-cryo. [1,6] Use of these compounds and mixtures, the behavior of which in the vapor phase is well understood, enabled us to test the portable apparatus with a variety of test beds and matrices and to compare the results to those previously obtained with the laboratory-based PLOT-cryo approach.

Finally, we explored the sensitivity limits of the portable PLOT-cryo unit when applied to diesel fuel, a complex mixture of common concern for environmental reasons. This was done on two matrices: glass beads and soil.

\subsection{Materials and Methods}

For all analyses presented in this paper, we used PLOT capillary wafers comprised of single or multiple alumina coated PLOT capillaries for vapor collection. During each experiment, the capillary wafer was cooled during vapor collection and heated during elution. The test beds were sampled at ambient room temperature. The borosilicate glass beads were obtained from a commercial supplier, and were typical chromatographic glass beads. For the work on coumarin and TNT, nominal $1 \mathrm{~mm}$ beads were used (which measured $1.2 \pm 0.05 \mathrm{~mm}$ ). For the work on diesel fuel, a 50/50 ( $\mathrm{vol} / \mathrm{vol}$ ) mixture of 1 - and $2 \mathrm{~mm}$ nominal beads were used. The $1 \mathrm{~mm}$ nominal beads were of the same diameter as those used for the coumarin and TNT; the $2 \mathrm{~mm}$ nominal beads measured $1.8 \pm 0.2 \mathrm{~mm}$. The soil used in the work on diesel fuel was clay soil collected locally on the NIST Boulder campus, and air-dried in our lab for three years. The soil was then size-selected for particles between 1.7 and $2.0 \mathrm{~mm}$ in diameter using standard testing sieves (nominal grating numbers 10 and 12). This prepared soil was then used in the manner described in section 3.4. The specifics on each solute will be presented later in the discussion of the results.

Spectroscopic-grade acetone was used as the solvent for all capillary elutions. The acetone was analyzed in our lab using established GC-MS protocols. [7] We chose this solvent because it is an effective eluent when paired with alumina coated capillaries, and for the safety of the researchers, especially student workers. The capillaries were eluted with acetone into crimp-cap autosampler vials and analyzed using gas chromatography coupled with either single-quadrupole mass spectrometry (GC-MS) or tandem quadrupole - time of flight mass spectrometry (GCQTOF) operated in single MS mode with high mass resolution.

\subsection{Experimental Details and Results}

For context and clarity, the experimental details are combined with the results in the following sections. 


\subsection{Initial Studies: Coumarin and TNT}

As with the laboratory implementation of PLOT-cryo, the first demonstrations of the field portable PLOT-cryo vapor sampling apparatus were done with coumarin dispersed on glass beads and TNT dispersed on glass beads. [6] The sample test beds for these solutes were $25 \mathrm{~mL}$ scintillation vials equipped with plastic caps, into which two small holes (an inlet and an outlet, both of which were $3.2 \mathrm{~mm}, 0.125 \mathrm{in}$ ) were drilled. Such test beds have been used and described previously. [6] In this work, approximately $50 \mathrm{mg}$ of solid coumarin and solid TNT were solvent dispersed (in acetone) separately on approximately $5 \mathrm{~g}$ of $2 \mathrm{~mm}$ glass beads. Subsequent to deposition, the solvent was slowly evaporated in a vacuum desiccator. The vapor collection from the vials of these compounds was done with the field portable PLOT-cryo apparatus described fully in Part I.[1] Multiple experiments were conducted to demonstrate both the hand piece and the standoff module and probe, in combination with both the single and multiple capillary wafers. For a typical test, vapor collection was allowed to proceed for 3 minutes with the sample at laboratory ambient temperature $\left(23 \pm 1^{\circ} \mathrm{C}\right)$, while the PLOT capillary was chilled to $-20 \pm 5{ }^{\circ} \mathrm{C}$. Chilling was done with the cold air vortex tube on the portable apparatus. Following solute collection, the PLOT wafers were then heated to $80 \pm 5{ }^{\circ} \mathrm{C}$ with the hot air vortex tube. Once warmed, the wafer was eluted with $1.5 \mathrm{~mL}$ of acetone for each vapor collection trial, and the resulting solutions were analyzed by gas chromatography with (single quadrupole) mass spectrometry (GC-MS).

For coumarin, the following GC-MS method was used: $30 \mathrm{~m}, 5$ percent phenyl polydimethylsiloxane column, coating thickness of $0.1 \mu \mathrm{m}$; splitless injection via automatic sampler, temperature of injector $=325^{\circ} \mathrm{C}$ at $55 \mathrm{kPa}(8 \mathrm{psi})$ constant head pressure; injection volume $=3 \mu \mathrm{L}$, column temperature of $55^{\circ} \mathrm{C}$ for $0.75 \mathrm{~min}$, followed by a temperature program at $99^{\circ} \mathrm{C} / \mathrm{min}$ to $80^{\circ} \mathrm{C}$, then $35^{\circ} \mathrm{C} / \mathrm{min}$ to $275^{\circ} \mathrm{C}$; scan $\mathrm{m} / \mathrm{z}$ from 35 to 350, SIM m/z $=89,90,118$, 146. For TNT, the following method was used with the same column: splitless injection via automatic sampler, temperature of injector $=275^{\circ} \mathrm{C}$ at $83 \mathrm{kPa}(12 \mathrm{psi})$ head pressure; injection volume $=3 \mu \mathrm{L}$, column temperature of $60^{\circ} \mathrm{C}$ for $1 \mathrm{~min}$, followed by a temperature program at 80 ${ }^{\circ} \mathrm{C} / \mathrm{min}$ to $170{ }^{\circ} \mathrm{C}$, then $35{ }^{\circ} \mathrm{C} / \mathrm{min}$ to $290{ }^{\circ} \mathrm{C}$; scan $\mathrm{m} / \mathrm{z}$ from 35 to $550, \mathrm{SIM} \mathrm{m} / \mathrm{z}=89,134,180$, 210. [7, 8] The scan mode allows more facile library matching for identification (where the libraries themselves contain scan-mode data), while the SIM mode is more sensitive and especially useful for a definitive go or no-go that the compound was detected in the vapor. [9]

All the experiments were successful in identifying the target compounds with the single and multiple capillary wafers, in the standoff module and with the handpiece; for brevity we will discuss the results obtained using a multiple capillary wafer and the standoff module. Typical sample chromatograms are provided in Figure 1 (a-d). The target analyte was easily detected with excellent signal-to-noise ratios in both scan and SIM modes. The recovery obtained by use of the multiple capillary wafer and the standoff probe was as good as that achieved in the laboratory. Earlier, for example, we determined that we could detect and quantitate TNT in the vapor above a substrate with $0.064 \mu \mathrm{g} / \mathrm{g}$ TNT (repeatability of 10\% COV) on the glass bead matrix. [3] The major differences in this work (as reflected in Figure 1, a-d) as compared with the earlier laboratory work (also done with vials as the test beds) are the collection speeds and the fact that all collections are done with the sample at ambient temperature. The much higher flow rate (with no loss of surface contact) provided by the multiple capillary wafer makes faster collection possible. This is in sharp contrast with the early laboratory work in which the coumarin samples were collected at $1 \mathrm{~mL} / \mathrm{min}$ for $60 \mathrm{~min}$ at a sample temperature of $110^{\circ} \mathrm{C}$, and TNT was collected at $1 \mathrm{~mL} / \mathrm{min}$ for $120 \mathrm{~min}$ at a sample temperature of $125^{\circ} \mathrm{C}$. Furthermore, the collection of TNT vapor was conducted at near ambient temperature $\left(30{ }^{\circ} \mathrm{C}\right)$; in the previous 
work, collection at this temperature at $1 \mathrm{~mL} / \mathrm{min}$ required $4000 \mathrm{~min}$ (nearly 3 days) with a single capillary. [6] The long collection times required in the earlier measurements caused the decomposition and discoloration of the dispersed TNT. Discoloration is nearly always noted with this solute when it is exposed for sampling for more than a few minutes. No such discoloration was noted in this work; the vapor collection was completed rapidly enough to avoid this commonly observed color change.

\subsection{Aviation Turbine Kerosene}

Because the multiple capillary wafer is especially suited for larger sample volumes, $118 \mathrm{~mL}$ (4 oz) paint cans were also used as test beds. These provided samples of a larger size to better simulate field conditions. As with the vials, two small holes (an inlet and an outlet, both of which were $3.2 \mathrm{~mm}, 0.125 \mathrm{in}$ ) were drilled in the lid of the paint cans. The solutes tested with this approach have included aviation turbine kerosene (JP-5, a specially formulated low volatility gas turbine kerosene used extensively on aircraft carriers), crude oil and a selection of natural essential oils. In each case, one drop of fluid was placed in the paint can, and the lid affixed. After about 1 hour of equilibration time, the standoff probe was inserted into one of the holes, and vapor was collected for $30 \mathrm{~s}$ by use of the multiple capillary wafer. Collection was done with the sample at laboratory ambient temperature $\left(23 \pm 1^{\circ} \mathrm{C}\right)$, while the capillary wafer was chilled to $-20 \pm 5{ }^{\circ} \mathrm{C}$. The PLOT wafer was then heated to $80 \pm 5^{\circ} \mathrm{C}$ and eluted with $1.5 \mathrm{~mL}$ of acetone for each vapor collection trial. For brevity in this article, only the results for JP-5 are presented. JP-5 is a well-characterized fuel, the vapor phase of which was previously studied using the advanced distillation curve method. [10]

For JP-5, the following GC-MS method was used to analyze the eluted sample: $30 \mathrm{~m}$, 5 percent phenyl polydimethylsiloxane column with a coating thickness of $0.1 \mu \mathrm{m}$; splitless injection via automatic sampler, temperature of injector $=325^{\circ} \mathrm{C}$ at $83 \mathrm{kPa}(12 \mathrm{psi})$ head pressure, injection volume $=2 \mu \mathrm{L}$, column temperature of $50{ }^{\circ} \mathrm{C}$ for $2 \mathrm{~min}$, followed by temperature program at 10 ${ }^{\circ} \mathrm{C} / \mathrm{min}$ to $150{ }^{\circ} \mathrm{C}$, then $60{ }^{\circ} \mathrm{C} / \mathrm{min}$ to $325^{\circ} \mathrm{C}$; hold for $1 \mathrm{~min}$; scan m/z from 15 to $550, \mathrm{SIM} \mathrm{m} / \mathrm{z}$ $=51,55,56,57,65,71,77,84,85,91,96,99,105,106,109,112,113,114,115,116,117,118$, $119,121,124,128,131,142,156,169,183,198,212,226$, and 240. The large number of ions used for the kerosene reflects the very complex chromatogram typically obtained for such fluids. The chromatograms are shown in Figure 2a and $2 \mathrm{~b}$. While the SIM chromatogram is simpler, the use of fewer ions spread over the $\mathrm{m} / \mathrm{z}$ range does not provide a sensitivity enhancement; however, the noise is reduced. The chromatogram of the collected headspace allows a reliable identification of the sample on the basis of the composition. Indeed, it is possible to readily distinguish the sample as a relatively low volatility aviation turbine kerosene from the chromatogram by comparison with known samples. [10]

\subsection{Naphthalene}

The utility of the multiple capillary wafer in rapid sampling for solutes dispersed in even larger volumes was illustrated by use of a solute diffuser (similar to a scent or odorant diffuser) to distribute solute in a closed environment or chamber. The solute diffuser was made from one of the aforementioned $118 \mathrm{~mL}$ cans, prepared by drilling a circular pattern of $0.3175 \mathrm{~cm}(0.125 \mathrm{in})$ holes in the lid. Then, $50 \mathrm{mg}$ of naphthalene was placed into the can and the lid firmly affixed. This can was placed in a hard sided valise $(40 \times 20 \times 20 \mathrm{~cm}, 16 \times 8 \times 8$ in) into the side of which a small hole was drilled. The diffuser was allowed to equilibrate inside the valise for $4 \mathrm{~h}$ at ambient temperature $\left(23{ }^{\circ} \mathrm{C}\right.$ with a variation of $\left.1^{\circ} \mathrm{C}\right)$. One can approximate the concentration of naphthalene in the vapor phase in the valise based on the partial pressure of naphthalene. Assuming that air and naphthalene behave as ideal gases, at equilibrium approximately $9.5 \mathrm{X}$ $10^{-5} \mathrm{~mol}$ of vapor will be present, resulting in a concentration of $0.07 \%$ (mass/mass). Note that 
this is an upper bound subject to the efficiency of the solute diffuser; the actual concentration is likely lower due to incomplete equilibration, which was not measured in this work. The vapor inside of the valise was then sampled by use of the standoff probe and multiple capillary wafer that was chilled to $-10 \pm 5{ }^{\circ} \mathrm{C}$. In these tests, sampling was allowed to proceed for $3 \mathrm{~s}$. The wafers were then heated to $60 \pm 5^{\circ} \mathrm{C}$ and eluted with $1.5 \mathrm{~mL}$ of acetone.

The eluted naphthalene sample was analyzed by mass spectrometry with the following method: $30 \mathrm{~m}, 5$ percent phenyl polydimethylsiloxane column with a coating thickness of $0.1 \mu \mathrm{m}$; splitless injection via automatic sampler, temperature of injector $=275^{\circ} \mathrm{C}$ at $83 \mathrm{kPa}(12 \mathrm{psi})$ head pressure, injection volume $=1 \mu \mathrm{L}$, column temperature of $50^{\circ} \mathrm{C}$ for $2 \mathrm{~min}$, followed by temperature program at $60{ }^{\circ} \mathrm{C} / \mathrm{min}$ to $100{ }^{\circ} \mathrm{C}$, then $20^{\circ} \mathrm{C} / \mathrm{min}$ to $175^{\circ} \mathrm{C}$; hold for $1 \mathrm{~min}$; scan m/z from 33 to $330, \mathrm{SIM} \mathrm{m} / \mathrm{z}=125$. The chromatogram measured in scan mode is provided in Figure $3 \mathrm{a}$, while the SIM is provided in $3 \mathrm{~b}$. In both chromatograms, the naphthalene elutes at approximately 6 min. In scan mode, the mass spectrum allows easy identification via a library search (although the early part of the chromatogram shows the noise of an incompletely cleaned multiple capillary wafer). The SIM trace is striking in that it shows an intense peak in response to the target compound; the fact that it is the result of only a 3 s collection demonstrates the practical utility of the multiple capillary wafer. Indeed, even shorter sampling times are possible for field applications. We do not report shorter collection times here because of difficulty in measuring such shorter collection times reliably. The ability to rapidly sample the vapor and efficiently desorb solute for analysis makes the approach well suited to sampling large volumes.

\subsection{Diesel fuel on soil}

For this demonstration of one environmental application of the field portable apparatus, a typical diesel fuel was spiked onto clay soil described in section 2.0. The diesel fuel was a commercial winter-grade, low-wax, ultralow-sulfur formulation that was refined from petroleum of the Denver-Julesburg field, and incorporated with a red dye indicating off road use. This loweraromatic fuel typically has a cetane number between 51 and 52 . This fluid was stored at $7{ }^{\circ} \mathrm{C}$ to preserve any volatile components, and no phase separation was observed. The fluid was chosen in part because it has been well-characterized in previous work. [11] One drop of this diesel fuel was dissolved in a scintillation vial of acetone. In the $118 \mathrm{~mL}$ paint can test bed, we prepared clay soil mixtures spiked with the diesel fuel/acetone mixture to obtain samples with concentrations $40 \mathrm{ppm}, 8.7 \mathrm{ppm}$, and 4 ppm (mass/mass). To obtain the lower-concentration samples, we serially diluted the appropriate mass of spiked soil into clean soil. Samples were mixed well in gas-tight earthen jars on a rolling mill for 1 hour.

The vapor collection from the cans was done using the portable unit's standoff module. We modified the paint can test bed by addition of a wire mesh screen, attached to the lid of the can on the inside of the can. This shielded the standoff probe from direct contact with the soil, as this would contaminate the vapor analysis. The sample collection temperature was ambient $(23 \pm$ $1{ }^{\circ} \mathrm{C}$.) The multiple capillary wafer was cooled and maintained at $-10 \pm 5{ }^{\circ} \mathrm{C}$ during collection. Collection was allowed to proceed for 10 minutes; subsequently the wafer was heated (to $60 \pm$ $5^{\circ} \mathrm{C}$ ) and eluted with $1.5 \mathrm{~mL}$ acetone.

Analysis of the eluted samples was done by GC-MS as described earlier. The following program was used: $30 \mathrm{~m}, 5$ percent phenyl methyl siloxane column with a coating thickness of $0.25 \mu \mathrm{m}$; splitless injection via automatic sampler; temperature of injector $=325^{\circ} \mathrm{C}$ at 10 psi head pressure; injection volume $=3 \mu \mathrm{L}$, column temperature of $33^{\circ} \mathrm{C}$ for $1 \mathrm{~min}$, followed by temperature program at $70{ }^{\circ} \mathrm{C} / \mathrm{min}$ to $80{ }^{\circ} \mathrm{C}$, then $35^{\circ} \mathrm{C} / \mathrm{min}$ to $170{ }^{\circ} \mathrm{C}$; hold for $2 \mathrm{~min}$; $\mathrm{SIM} \mathrm{m} / \mathrm{z}$ $=41,42,43,44,53,55,56,57,58,67,69,70,71,81,82,83,84,85,97,98,99,113$, and 142 . 
Diesel fuel contains hundreds of components, and during early experiments, we identified one high-abundance component in the vapor phase, n-decane, that could serve as a sentinel compound indicating the presence of diesel fuel on soil. An example chromatogram measured in SIM mode is provided in Figure 4.

More dilute preparations of diesel fuel on soil were analyzed until the detection limit, $4 \mathrm{ppm}$ (mass/mass), was determined by assessment of the n-decane signal relative to noise. We selected a minimum acceptable signal-to-noise ratio of $3: 1$.

This analysis was challenging because of the high degree of complexity in the diesel fuel mixture. Even utilizing SIM mode, the ions of interest for n-decane were shared by other hydrocarbon compounds that neighbored or co-eluted with the n-decane peak. We identified the unit-mass resolution of the single quadrupole MS as a limitation in this work. In the following section, we apply an accepted detection limit determination method using the more sensitive QTOF mass spectrometer.

\subsection{Sensitivity assessment: Diesel fuel on glass beads}

It was of interest to us to determine at what level the portable apparatus is effective at detecting a typical analyte when GC-MS was used. We used the EPA standard, Appendix B to Part 136, Definition and Procedure for the Determination of the Method Detection Limit, for the determination of the method detection limit. [12] This guidance was developed specifically to be sensitive to matrix, instrument, and analyst effects, and captures the uncertainty inherent in sample collection, processing, and analysis. We chose the EPA standard because it enabled us to include the real-world sources of variability inherent with this type of portable method applied to complex mixtures. In the guideline, the method detection limit (MDL) is calculated in terms of signal units (in our case, area counts under the mass spectral peak) by multiplying the sample standard deviation of at least seven samples of the same concentration and preparation(s) by the Student's t-value for the corresponding degrees of freedom and desired confidence level. We selected alpha $=0.05$ for this calculation.

$$
\mathrm{MDL}=\mathrm{s} * \mathrm{t}_{\mathrm{n}-1,0.05}
$$

For this assessment of its sensitivity, we selected diesel fuel on glass beads as the test analyte. We chose a spike level of $1 \mathrm{ppm}$ (mass/mass) diesel fuel for our replicate preparation based on preliminary experiments exploring the method's sensitivity at varying levels. We employed a laboratory preparation using the same diesel fuel described in section 3.4; however, in these experiments this fluid was spiked onto a matrix of a 50/50 (vol/vol) mixture of 1 and $2 \mathrm{~mm}$ (nominal) borosilicate glass beads in the $118 \mathrm{~mL}$ paint can test bed. Preparations of the diesel fuel and glass beads ( $1 \mathrm{ppm}$ by mass) were prepared in clean paint cans by serial dilution. Higher concentration samples were prepared $(40 \pm 10 \mathrm{ppm})$ by placing drops of diesel fuel into cans filled with glass beads (270 $\pm 30 \mathrm{~g}$ ). The lids were affixed, and the sealed cans were mixed on a rolling mill for 45 minutes to ensure thorough distribution of the solute. Solvent dispersion was not used in these experiments. Subsequently, sixteen replicate mixtures $(1 \pm 0.0365 \mathrm{ppm})$ were prepared by placing the appropriate amounts $(20 \pm 10 \mathrm{~g})$ of the higher-concentration spiked samples into additional cans with clean glass beads $(260 \pm 30 \mathrm{~g})$ to achieve the desired final concentration. The cans were sealed and mixed as above and sampled using the field portable apparatus the same day.

The vapor collection from each can was carried out at ambient temperature using the standoff module with the wafer cooled to $-10{ }^{\circ} \mathrm{C}\left( \pm 5^{\circ} \mathrm{C}\right)$. Collection was allowed to proceed for 10 minutes for these tests. This is longer than what might be desirable for field use; however, we 
chose this sampling time as a benchmark of optimized performance. After vapor collection, the wafer was then heated to $60^{\circ} \mathrm{C}\left( \pm 5^{\circ} \mathrm{C}\right)$ and eluted with $1.5 \mathrm{~mL}$ acetone.

Contamination is a concern whenever analyzing samples near the detection limit of a method. Frequent rinsing and careful handling of the entire apparatus was required to minimize residual contamination from previous runs. We tested the components of the apparatus regularly for cleanliness and ran daily matrix blanks on the glass beads to establish background signal and ensure there was no carryover.

Eluted samples were analyzed using GC-QTOF mass spectrometry. Time-of-flight (TOF) mass analyzers are capable of very high resolution and mass accuracy, and are therefore powerful tools in the identification of unknown components. Our instrument maintained sub-ppm mass accuracy and resolution of about 7000 in our $\mathrm{m} / \mathrm{z}$ range of interest during these measurements, which enabled us to identify components assignable to diesel fuel to their unequivocal chemical formulas, given only their parent ion mass-to-charge ratio. We note that although our instrument is capable of tandem MS/MS, for these measurements, the quadrupole was operated in full transmission mode, and the collision cell was not in use. The following method was used: $30 \mathrm{~m}$, 5 percent phenyldimethylsiloxane column with a coating thickness of $0.25 \mu \mathrm{m}$; pulsed split injection $\left(15: 1 ; 25\right.$ psi pulse) via automatic sampler; temperature of injector $=300{ }^{\circ} \mathrm{C}$ at 8 psi head pressure; injection volume $=1 \mu \mathrm{L}$, column temperature of $50^{\circ} \mathrm{C}$ for $1 \mathrm{~min}$, followed by temperature program at $20^{\circ} \mathrm{C} / \mathrm{min}$ to $120^{\circ} \mathrm{C}$, then $50{ }^{\circ} \mathrm{C} / \mathrm{min}$ to $280{ }^{\circ} \mathrm{C}$; hold for $2 \mathrm{~min}$; emission current $=35 \mathrm{~mA}$; scan m/z from 50 to $300 ; 200 \mathrm{~ms} / \mathrm{scan}$. Each eluted sample was run in triplicate and the responses averaged.

We used the same single-compound approach as in section 3.4; however, in this experiment we identified a compound eluting around $4.9 \mathrm{~min}$, with the chemical formula $\mathrm{C}_{9} \mathrm{H}_{12}$, as the sentinel indicating the presence of diesel fuel. We found this component to be much more abundant than $\mathrm{n}$-decane in the headspace of these samples; therefore, we replaced n-decane (used in the previous section) as the sentinel compound for the calculation of the method detection limit. Differences between the matrices of soil and glass beads is one probable reason for this difference in abundance in the vapor. Using the high mass accuracy of the QTOF described above, we determined that only hydrocarbon compounds with this chemical formula could yield the parent ion mass $120.0939 \mathrm{~kg} / \mathrm{kmol}(120.0939 \mathrm{amu})$. Diesel fuel contains several components with this chemical formula, and through retention time matching and analysis of the fragmentation pattern, we identified the compound as 1,2,4-trimethylbenzene (TMB). TMB is an appropriate marker to use when analyzing diesel fuel for several reasons. Because of its aromaticity and relatively high volatility, TMB is present in high concentrations in the headspace. It is also one of the more carefully-regulated chemicals present with high abundance in diesel fuel, which demonstrates the applicability of this technology in regulatory situations. TMB is regulated for occupational exposure by the National Institute for Occupational Safety and Health (with a threshold limit value, TLV, of $25 \mathrm{ppm}$ ), the American Conference of Governmental Industrial Hygienists (25 ppm), and the US Environmental Protection Agency (45 ppm). [13-15] Additionally, current EPA Superfund protocols for site inspections are limited to sample collection at the surface of the suspected polluted area. [16] One of the advantages of the field portable PLOT-cryo apparatus is that, with the standoff probe attachment, chemical information can be safely and effectively derived from areas well beneath the soil's surface. One of our goals in performing the method detection limit calculation was to confirm that the portable PLOT-cryo method performs with sufficient sensitivity to be applied in these types of regulatory situations.

For each of the sixteen $1 \mathrm{ppm}$ replicates, we generated extracted ion chromatograms for the exact masses 105.0767 and 120.0939 (mass accuracy 200 ppm). An example of these is shown in 
Figure 5. The $120.0939 \mathrm{~m} / \mathrm{z}$ ion was used to confirm the identity of the peak. We used the signal generated by the $105.0767 \mathrm{~m} / \mathrm{z}$ ion in our MDL calculations because it was the more abundant fragment.

The sample standard deviation for the sixteen 1 ppm replicates was 15194 arbitrary units (AU). The appropriate Student's t-value for 15 degrees of freedom, with a confidence interval of $95 \%$, is 2.131. The calculated MDL was 32378 AU. This represents the area count or signal threshold above which diesel fuel contamination can be detected $95 \%$ of the time. The MDL reported here is strictly applicable to diesel fuel spiked on a matrix of glass beads, sampled with the field portable apparatus, eluted with $1.5 \mathrm{~mL}$ of solvent, and analyzed using the GC-QTOF method described above. We did not attempt quantitation based on instrument response; however, based on the mean signal obtained from the $1 \mathrm{ppm}$ replicates (53997 AU), the signal detection limit we obtained corresponds to an MDL below 1 ppm.

We observed a great deal of variation in the signal for replicates of the same concentration, evidenced by the sample standard deviation. Even for samples collected at much higher concentrations during preliminary experiments, we were unable to satisfactorily calibrate the signal and quantitate the sentinel compound. We would like to note that this variation was a result of sampling uncertainty between separately-prepared $1 \mathrm{ppm}$ samples; it was not a result of the instrument's repeatability.

The most likely source of this variation is the sampling flow rate. We did not monitor the suction flow rate during collection for every sample, but during routine checks, we observed rates varying between 55 and $72 \mathrm{~mL} / \mathrm{min}$ (a decrease from $180 \mathrm{~mL} / \mathrm{min}$ observed with the newly fabricated unit discussed in Part I). [1] The high variability in flow rate for the field portable apparatus can be ascribed to two major sources. First, there is a great deal of variability in the suction (vacuum) that is generated by the laboratory compressed air. While the flow of compressed air to the vacuum generator is controlled by a valve, the pressure of the "house air" is not controlled at the portable unit. Variations in the pressure and flow rate occur as a result of intermittent and random compressed air usage elsewhere in the building. Clearly, under such circumstances, the pressure is not controlled as precisely as the electronic pressure control inlets used in the laboratory implementation of PLOT-cryo, for example. The compressed air source is used in the operation of both the heating and cooling vortex tubes as well as the suction, further adding to the variability in the proportion of compressed air pressure available to the vacuum generator. The second major factor in variability is flow rate impedance caused by the matrix geometry. Because the standoff probe is positioned beneath the surface of the matrix during sample collection, the suction may cause matrix particles (in this case glass beads) to become lodged against the probe and thus partially impede flow rate. This can occur randomly, and is a vagary of any matrix (glass bead, soil, etc.)

A third major source of variation among samples is the unknown local vapor concentration in the test beds. The condensed-phase solute concentration on the glass beads was nominally $1 \mathrm{ppm}( \pm$ $0.037 \mathrm{ppm}$ ); however, differences in pressure, temperature, humidity, and solute distribution could affect the vapor concentration a great deal. It is also worth noting that, especially at such a low concentration, depletion of the analyte likely occurred both locally, in the matrix around the standoff probe, and in the whole volume of the test bed, which could affect the amount of solute in the vapor collected. Without addressing these issues, we cannot calibrate the method for quantitative measurements. This is not a serious disadvantage, however, since matrix variability will be present in nearly any field sampling scenario, and it was important for us to capture as much of this variability as possible. We are confident that our calculated MDL, 32378, corresponds to a limit in terms of concentration of below $1 \mathrm{ppm}$, given that the mean response to our $1 \mathrm{ppm}$ spiked samples was 53997. Future work will address control and monitoring of the flow rate and apply the method to known vapor concentrations to help attribute this variation to potential sources. 
421

422 


\subsection{Conclusions}

This work explored the applicability of the novel portable PLOT-cryo approach, discussed in Part I, to several benchmark analytes and a typical environmental situation. Our goals were to (1) confirm that the approach can effectively and reliably detect compounds present in the headspace above a condensed sample, and (2) calculate the method detection limit for a prototype complex sample, namely diesel fuel at a known concentration on a matrix of glass beads. We draw the following conclusions from this study:

(1) The field portable apparatus allowed for the successful collection and detection of the compounds in these experiments. We observed reliable results with samples maintained at ambient temperature and samples that were heated to promote mass transfer to the headspace. The method allowed for very short collection times (as low as $3 \mathrm{~s}$ ), enabled by the high flow rate through the multiple capillary wafer.

(2) Limit of detection was determined qualitatively by observing the chromatographic response to $4 \mathrm{ppm}$ diesel/soil when using a single quadrupole mass spectrometer. The MDL calculated for analyses done with QTOF was $32378 \mathrm{AU}$, the equivalent to a concentration below $1 \mathrm{ppm}$. The QTOF provided a significantly lower MDL than the single quadrupole instrument. By leveraging the high-mass-accuracy extracted ion counts achievable with QTOF detection, the sensitivity of the portable unit was significantly increased. According to the National Institute for Occupational Safety and Health, the acceptable exposure limit for trimethylbenzene is $25 \mathrm{ppm}$. [15] We expect, therefore, that the portable PLOT-cryo apparatus would reliably detect the conditions deemed problematic by federal regulations.

(3) We encountered issues with variability and contamination, especially when working at low concentrations, near the MDL. We attribute the increase in variability relative to results previously obtained with the laboratory PLOT-cryo apparatus to three major factors. These factors are inconsistency in the vacuum generator's source of compressed air, potential flow rate impedance due to matrix geometry, and local depletion of the solute around the probe. It should be noted that the matrix of glass beads, or a pure sample dispersed in an enclosed test bed, represents the least variable, "best" case. In real-world forensic or environmental samples, matrix effects and moisture levels would likely further increase variability.

(4) Quantitation has been successfully demonstrated with the lab-based PLOT-cryo approach in past work, but has not been achieved with the portable unit. [6] The laboratory apparatus achieves a better detection limit and lower variability than the portable unit, which we expected, given the challenges inherent in field sampling. Future development will focus on ways to improve performance. Studying samples with known vapor concentrations may yield more information about the precise sources of variability in sampling. 
465

466

467

468

469

470

471

472

\section{Acknowledgments}

MEH gratefully acknowledges a Professional Research Experience Program (PREP) Fellowship. SBR gratefully acknowledges a Summer Undergraduate Research Fellowship (SURF). All authors gratefully acknowledge the assistance of Dr. Tara M. Lovestead with the gas

chromatographic measurement of the eluted samples and the financial support of the NIST Office of Law Enforcement Standards. 


\section{Figure Captions}

Figure 1. Gas chromatograms showing typical analyses for vapor samples collected by use of the multiple capillary wafer from solutes dispersed on glass beads in scintillation vials, collected for 3 min. Panel (a) is for coumarin in scan mode, panel (b) is for coumarin in SIM mode; panel (c) is for TNT in scan mode and panel (d) is for TNT in SIM mode. Conditions are described in the text. The abundance is presented in arbitrary units.

Figure 2. Gas chromatograms showing typical analyses for vapor samples produced at ambient temperature from one drop of JP-5 (low volatility gas turbine kerosene) in a $118 \mathrm{~mL}$ (4 oz) paint can, collected for $30 \mathrm{~s}$. Panel a shows results from scan mode, panel b shows results from SIM. Conditions are described in the text.

Figure 3. Gas chromatograms showing analyses for vapor samples of $50 \mathrm{mg}$ naphthalene produced inside of a diffuser that was placed in a small valise. Vapor was collected by use of the multiple capillary wafer for $3 \mathrm{~s}$ at ambient temperature. Panel a shows results from scan mode, panel $b$ shows results from SIM. Conditions are described in the text.

Figure 4. A sample chromatogram in SIM mode showing the results of sampling a paint can containing a $4 \mathrm{ppm}$ mixture of diesel fuel and clay soil. An arrow points to the n-decane peak $(\mathrm{RT}=3.7 \mathrm{~min})$. Conditions are described in the text.

Figure 5. Sample extracted ion chromatograms showing the results of vapor samples of $1 \mathrm{ppm}$ diesel fuel on a matrix of glass beads. Panel a depicts the 1,2,4-trimethylbenzene base peak used to generate area counts (105.0767 amu, $200 \mathrm{ppm}$ mass accuracy). Panel b displays the parent ion (120.0939 amu \pm 200 ppm mass accuracy) peak overlaid with the supporting peak. The presence of both ions within an allowed relative abundance verified the identity of TMB. 


\section{References}

1. Bruno, T.J., Field Portable Low Temperature Porous Layer Open Tubular Cryoadsorption Headspace Sampling and Analysis Part I: Method and Apparatus. J Chromatog A. submitted.

2. Lovestead, T.M. and T.J. Bruno, Detection of poultry spoilage markers from headspace analysis with cryoadsorption on a short alumina PLOT column. Food Chem., 2010. 121: p. 1274-1282.

3. Lovestead, T.M. and T.J. Bruno, Trace Headspace Sampling for Quantitative Analysis of Explosives with Cryoadsorption on Short Alumina Porous Layer Open Tubular Columns. Anal. Chem., 2010. 82: p. 5621-5627.

4. Lovestead, T.M. and T.J. Bruno, Detecting gravesoil with headspace analysis with adsorption on short porous layer open tubular (PLOT) columns. Forens. Sci. Int., 2011. 204: p. 156-161.

5. Nichols, J.E., M.E. Harries, T.M. Lovestead, and T.J. Bruno, Analysis of arson fire debris by low temperature dynamic headspace adsorption PLOT columns. J. Chromatogr. A, 2013. 1334: p. 126-138.

6. Bruno, T.J., Simple, quantitative headspace analysis by cryoadsorption on a short alumina PLOT column. J. Chromatogr. Sci., 2009. 47: p. 5069-5074.

7. Bruno, T.J., Svoronos, P.D.N, CRC Handbook of Basic Tables for Chemical Analysis, 3rd. ed. . 2011, Boca Raton: Taylor and Francis CRC Press.

8. Bruno, T.J., Svoronos, P.D.N., CRC Handbook of Fundamental Spectroscopic Correlation Charts. 2006, Boca Raton: Taylor and Francis CRC Press.

9. MSDS, S-8 Synthetic Jet Fuel, material safety data sheet. Syntroleum Corporation, Tulsa, OK, 2005.

10. Burger, J.L. and T.J. Bruno, Application of the Advanced Distillation Curve Method to the Variability of Jet Fuels. Energy and Fuels, 2012. 26: p. 3661-3671.

11. Windom, B.C., M.L. Huber, T.J. Bruno, A.L. Lown, and C.T. Lira, Measurements and Modeling Study on a High-Aromatic Diesel Fuel. Energy and Fuels, 2012. 26: p. 1787-1797.

12. United States Code of Federal Regulations, Title 40: Protection of Environment, Part 136 Guidelines establishing test procedures for the analyses of pollutants, Appendix B to Part $136-$ definition and procedure for the determination of Method Detection Limit. 1999.

13. National Advisory Committee for Acute Exposure Guideline Levels for Hazardous Substances, Acute Exposure Guideline Levels (AEGLs) for 1,3,5-trimethylbenzene, 1,2,4-trimethylbenzene, 1,2,3-trimethylbenzene. 2007, US Environmental Protection Agency: Washington, DC.

14. American Conference of Governmental Industrial Hygienists, Trimethyl benzene isomers, in Documentation of the threshold limit values and biological exposure indices. 2002: Cincinnati, $\mathrm{OH}$.

15. National Institute for Occupational Safety and Health, NIOSH recommendations for occupational safety and health: Compendium of policy documents and statements. 1992, US Department of Health and Human Services: Cincinnati, $\mathrm{OH}$. 
16. Office of Emergency and Remedial Response, Guidance for Performing Site Inspections Under 


\section{FIGURES}
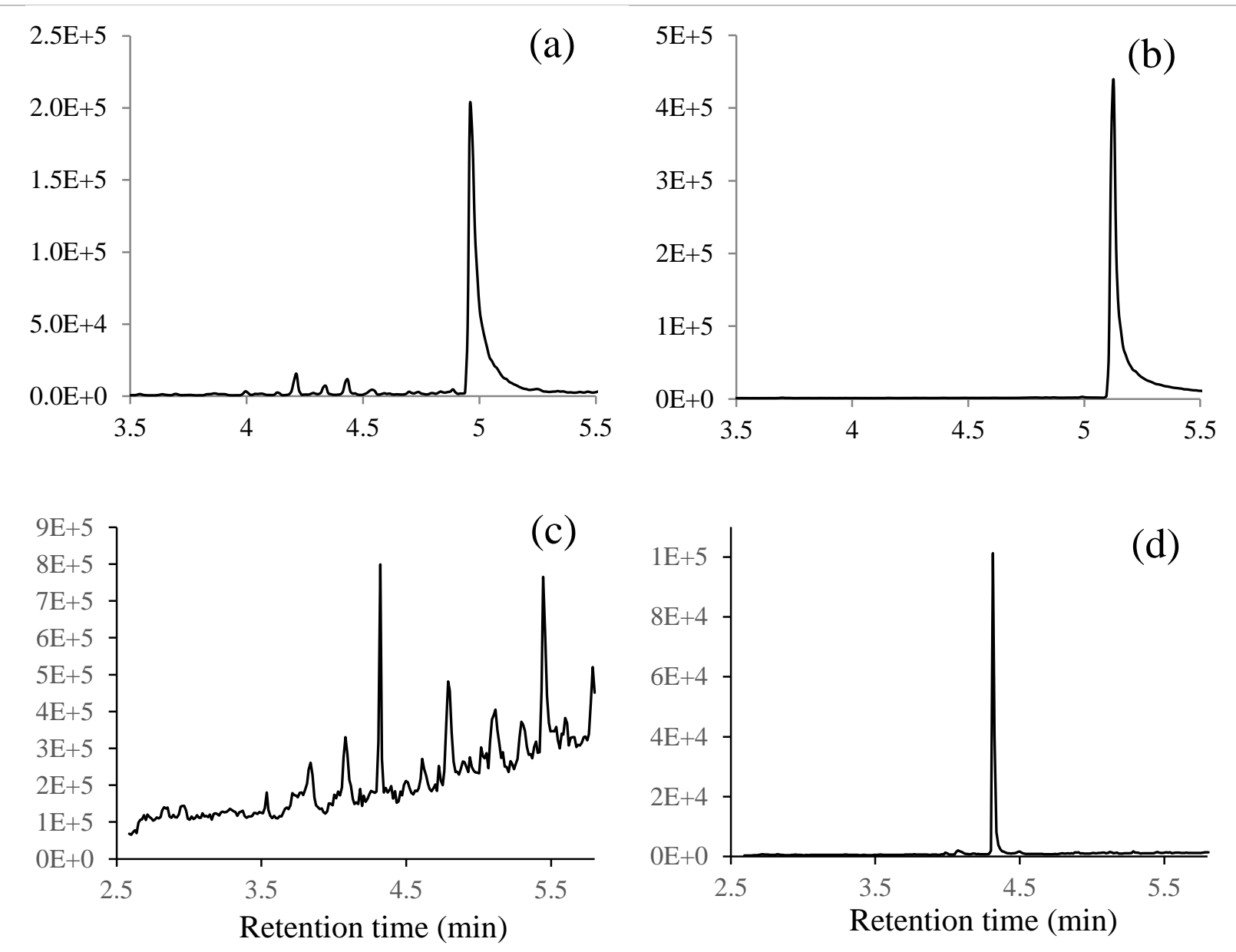

Fig 1 

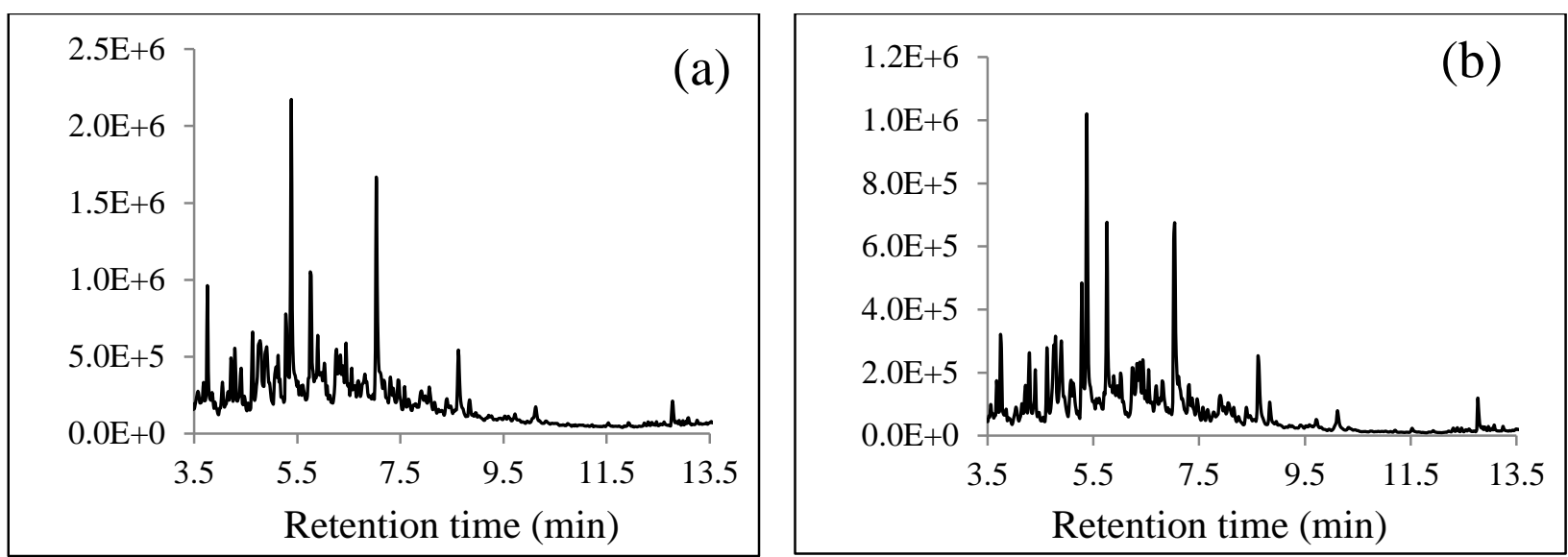

Fig 2 

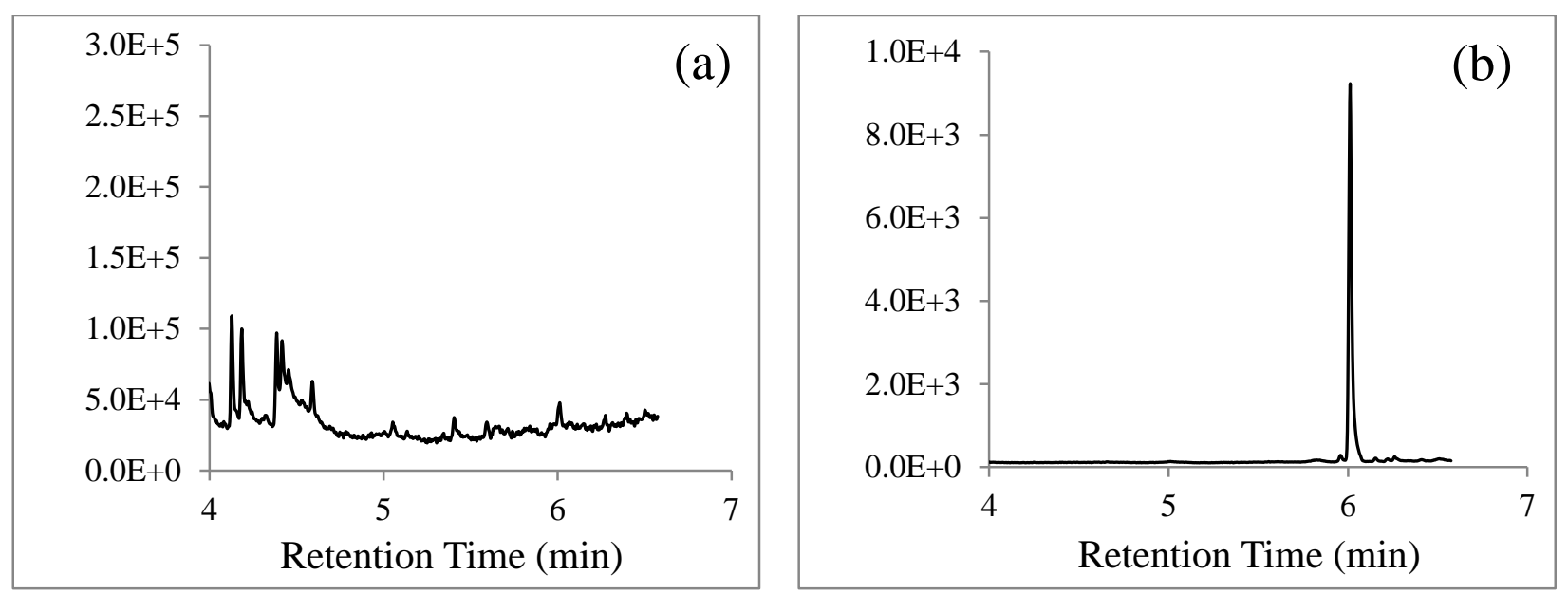

Fig 3 


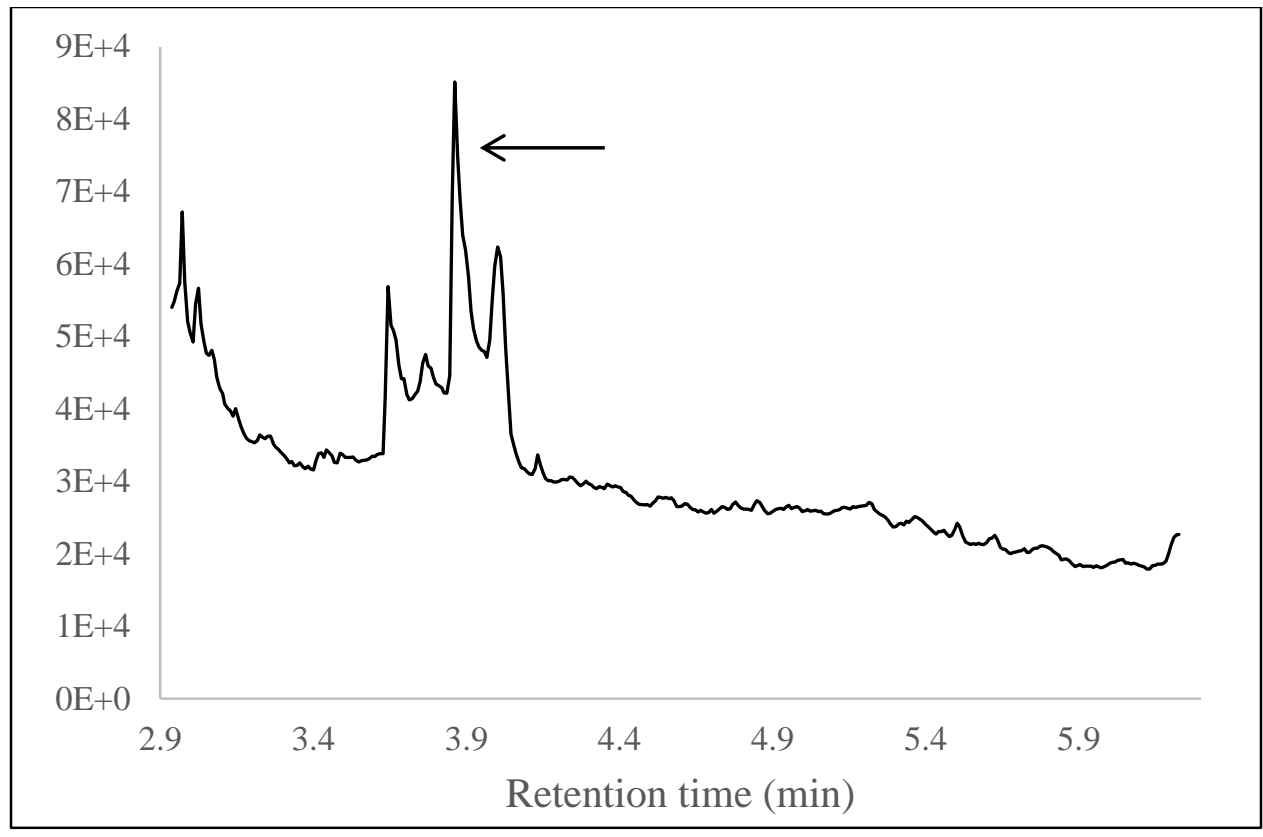

Fig 4 

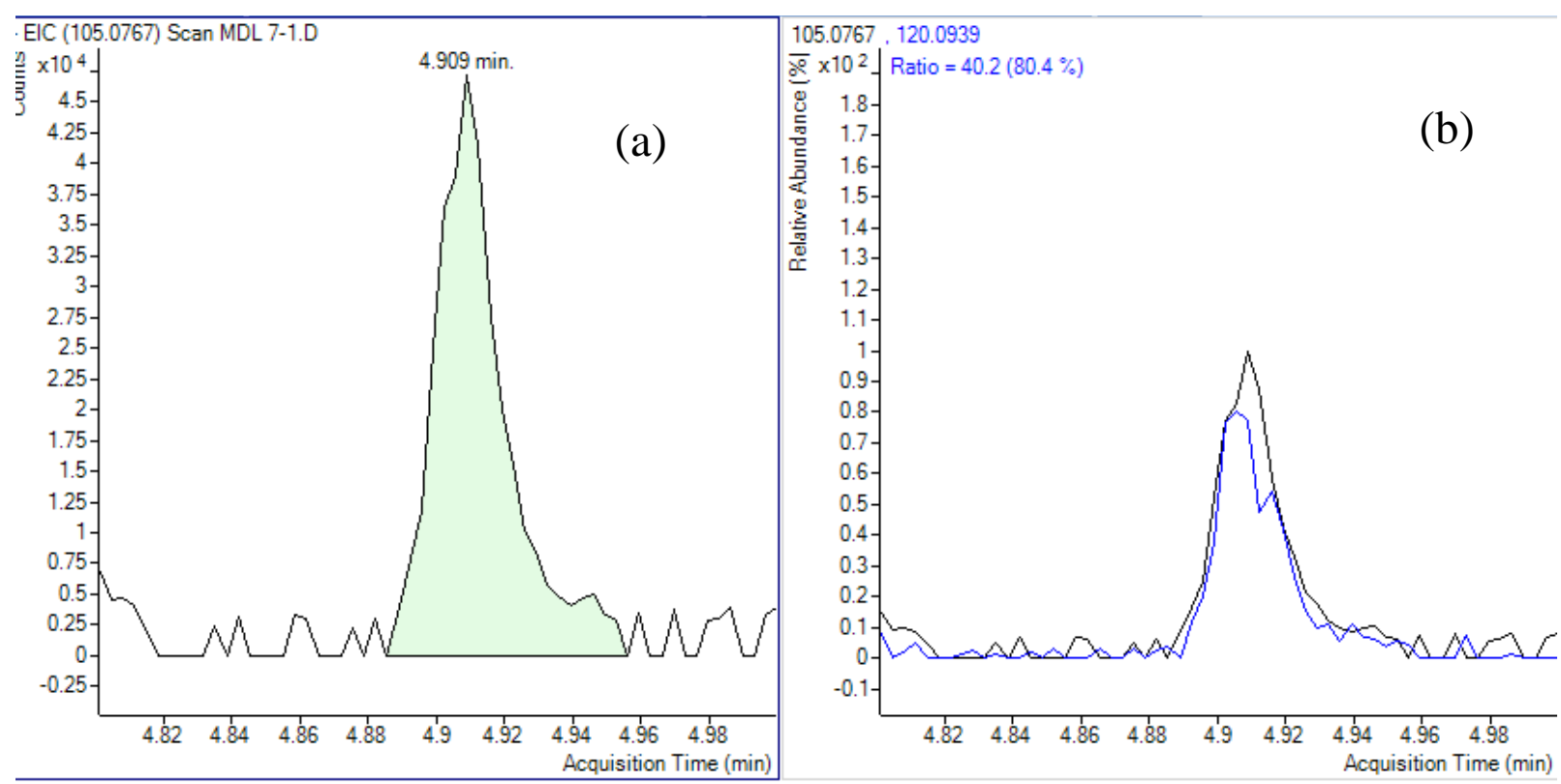

Fig 5 